\title{
WW domain-containing oxidoreductase promotes neuronal differentiation via negative regulation of glycogen synthase kinase $3 \beta$
}

\author{
H-Y Wang ${ }^{1,7}$, L-I Juo ${ }^{1,7}$, Y-T Lin ${ }^{2}$, M Hsiao ${ }^{3}$, J-T Lin ${ }^{4}$, C-H Tsai ${ }^{1}$, Y-H Tzeng ${ }^{1}$, Y-C Chuang ${ }^{5}$, N-S Chang ${ }^{6}$, C-N Yang ${ }^{\star, 5}$ and P-J Lu $^{\star, 1}$
}

WW domain-containing oxidoreductase (WWOX), a putative tumour suppressor, is suggested to be involved in the hyperphosphorylation of Alzheimer's Tau. Tau is a microtubule-associated protein that has an important role in microtubule assembly and stability. Glycogen synthase kinase $3 \beta$ (GSK3 $\beta$ ) has a vital role in Tau hyperphosphorylation at its microtubulebinding domains. Hyperphosphorylated Tau has a low affinity for microtubules, thus disrupting microtubule stability. Bioinformatics analysis indicated that WWOX contains two potential GSK3 $\beta$-binding FXXXLI/VXRLE motifs. Immunofluorescence, immunoprecipitation and molecular modelling showed that WWOX interacts physically with GSK3 $\beta$. We demonstrated biochemically that WWOX can bind directly to GSK3 $\beta$ through its short-chain alcohol dehydrogenase/reductase domain. Moreover, the overexpression of WWOX inhibited GSK3 $\beta$-stimulated S396 and S404 phosphorylation within the microtubule domains of Tau, indicating that WWOX is involved in regulating GSK3 $\beta$ activity in cells. WWOX repressed GSK3 $\beta$ activity, restored the microtubule assembly activity of Tau and promoted neurite outgrowth in SH-SY5Y cells. Conversely, RNAi-mediated knockdown of WWOX in retinoic acid (RA)-differentiated SH-SY5Y cells inhibited neurite outgrowth. These results suggest that WWOX is likely to be involved in regulating GSK3 $\beta$ activity, reducing the level of phosphorylated Tau, and subsequently promoting neurite outgrowth during neuron differentiation. In summary, our data reveal a novel mechanism by which WWOX promotes neuronal differentiation in response to RA.

Cell Death and Differentiation (2012) 19, 1049-1059; doi:10.1038/cdd.2011.188; published online 23 December 2011

WW domain-containing oxidoreductase (WWOX/WOX1/FOR) ${ }^{1}$ is a $46 \mathrm{kDa}$ protein that has been implicated in human tumourigenesis, nervous system development and Alzheimer's disease (AD). ${ }^{2-4}$ WWOX contains two N-terminal WW domains (containing conserved tryptophan residues) and one $\mathrm{COOH}$ terminal short-chain alcohol dehydrogenase/reductase (ADH/ SDR) domain. Both the WW and ADH domains mediate protein-protein interactions. The WWOX has been reported to bind to many proteins, including p53, p73, erythroblastic leukaemia viral oncogene homolog 4, ezrin, activator protein-2 $\gamma$, small integral membrane protein of the lysosome/late endosome, c-Jun N-terminal kinase 1 and mouse double mutant 2.,6 Although some studies have suggested that WWOX might have a critical role in regulating Tau hyperphosphorylation, ${ }^{2}$ the details of this regulation remain unclear.

Glycogen synthase kinase $3 \beta$ (GSK3 $\beta$ ), a serine/threonine kinase that was first identified based on its ability to phosphorylate glycogen synthase and regulate glycogen metabolism, is now known to be a key kinase in several important signalling pathways. The dysregulation of GSK3 $\beta$ is involved in several major human diseases, including diabetes, cancer, AD and bipolar disorder. ${ }^{7}$ Researchers have reported a number of proteins that associate with GSK3 $\beta$ and regulate its activity. The strongest examples is Axin, which are involved in the Wnt signalling pathway. ${ }^{2,8}$ Chou et al. ${ }^{9}$ used the yeast twohybrid method to identify a novel GSK3 $\beta$ interacting protein, GSKIP, which can bind to GSK3 $\beta$ and inhibit its kinase activity.

The role of GSK3 $\beta$ in regulating neural cell differentiation is controversial. GSK3 $\beta$ has been shown to facilitate neurite outgrowth by preventing E2F1 from inhibiting the transcription of the cell cycle inhibitors p21 and p15. ${ }^{10}$ However, other evidence has indicated that the inactivation of GSK3 $\beta$ results in collapsin response mediator protein 2 (CRMP-2) dephosphorylation, which leads to enhanced microtubule polymerisation and axon growth. ${ }^{11}$ Therefore, the function of GSK3 $\beta$ in the regulation of neural differentiation remains unclear.

Chen et al. ${ }^{12}$ reported that WWOX likely has a role in the development of the nervous system. They found that WWOX

\footnotetext{
${ }^{1}$ Institute of Clinical Medicine, National Cheng Kung University Medical College, Tainan, Taiwan; ${ }^{2}$ Neurology Section, Kaohsiung Veterans General Hospital, Kaohsiung, Taiwan; ${ }^{3}$ Genomics Research Center, Academia Sinica, Taipei, Taiwan; ${ }^{4}$ Urology Section, Department of Surgery, Kaohsiung Veterans General Hospital, Kaohsiung, Taiwan; ${ }^{5}$ Institute of Biotechnology, National University of Kaohsiung, Kaohsiung, Taiwan and ${ }^{6}$ Institute of Molecular Medicine, National Cheng Kung University Medical College, Tainan, Taiwan ${ }^{*}$ Corresponding authors: C-N Yang, Institute of Biotechnology, National University of Kaohsiung, Kaohsiung 700, Taiwan. Tel: +886 75979717 ; Fax: +886 73468207; E-mail: cnyang@nuk.edu.tw

or P-J Lu, Institute of Clinical Medicine, National Cheng Kung University Medical College, No. 35, Siaodong Road, Tainan 704, Taiwan. Tel: +886 62353535 extn. 4415 ; Fax: + 8866 2758781; E-mail: pjlu2190@mail.ncku.edu.tw

${ }^{7}$ These authors contributed equally to this work.

Keywords: SH-SY5Y; WWOX; GSK3 $\beta$; Tau

Abbreviations: RA, retinoic acid; WWOX, WW domain-containing oxidoreductase; GSK3 $\beta$, glycogen synthase kinase 3 3 ; ADH/SDR, short-chain alcohol dehydrogenase/reductase; GSKIP, glycogen synthase kinase $3 \beta$ interaction protein; CRMP-2, collapsin response mediator protein 2; MD, molecular dynamic; GST, glutathione $S$-transferase; GFP, green fluorescent protein; AD, Alzheimer's disease

Received 23.2.11; revised 09.11.11; accepted 10.11.11; Edited by M Deshmukh; published online 23.12.11
} 
was differentially expressed during various stages of brain development in mice, indicating a potential role for WWOX in promoting neuronal differentiation and maturation. ${ }^{12}$ However, the molecular mechanism by which WWOX facilitates neural differentiation has yet to be revealed. In this study, we demonstrated that WWOX enhances retinoic acid (RA)mediated neurite outgrowth and identified a novel mechanism through which WWOX promotes neurite outgrowth by interacting with and suppressing GSK3 $\beta$ kinase activity in the presence of RA. Our findings suggest that the regulation of GSK 3 activity by WWOX has a vital role in RA-induced neural-cell differentiation.

a
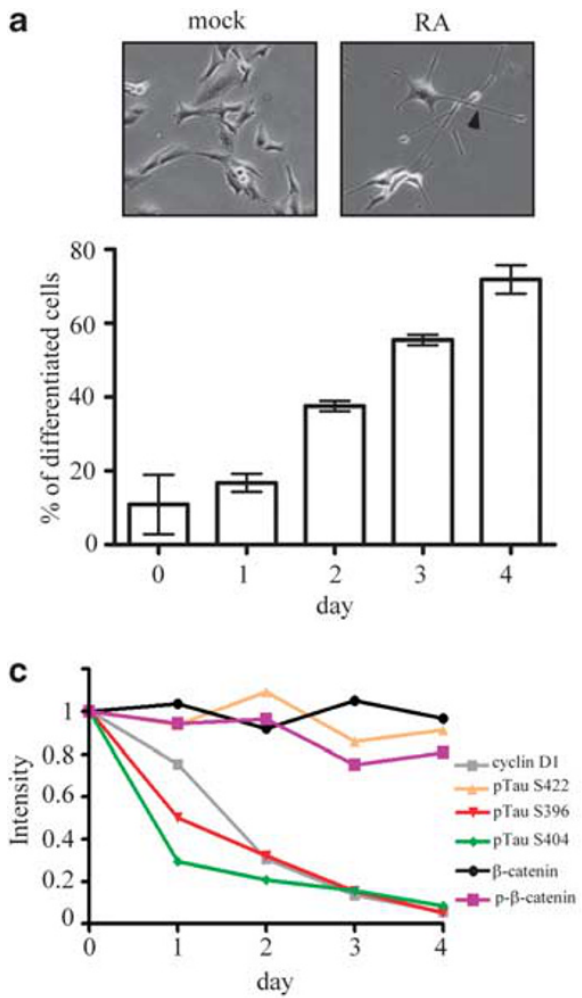

\section{Results}

WWOX is required for neuronal cell differentiation. RA can induce neuroblastomal differentiation in SH-SY5Y cells. ${ }^{13-15}$ From 1 to 4 days after RA induction, SH-SY5Y cells progressively underwent phenotypic changes that were compatible with neuron-like morphology, characterised by neurite outgrowth (Figure 1a). The expression of the neuronal marker $\beta$ III-tubulin progressively increased over 4 days of RA treatment (Figure 1b). The expression of cyclin D1, a cell-cycle progression marker, gradually decreased during the RA treatment. Increased expression of WWOX
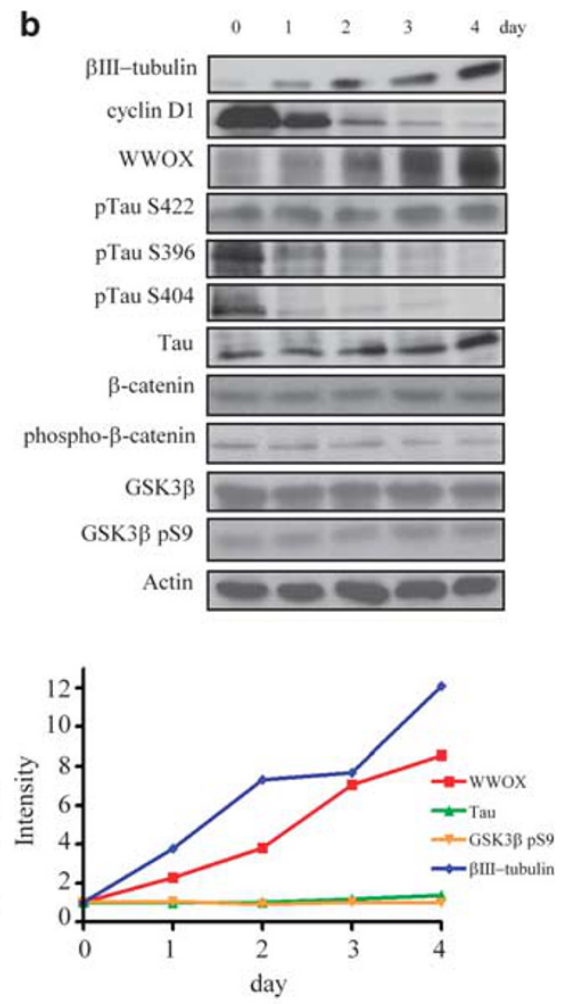

d

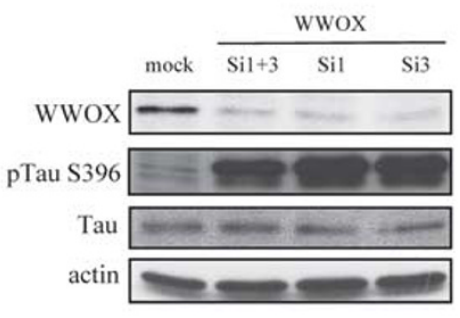

Figure 1 RA-induced SH-SY5Y differentiation is reduced by WWOX knockdown. (a) Morphological changes in SH-SY5Y neuroblastoma cells treated with or without RA (upper panel). The percentage of differentiated cells was determined for cells treated with $10 \mu \mathrm{M} \mathrm{RA}$, and the photos were taken on days $0,1,2,3$ and 4 (lower panel). (b) Cells were treated with RA for the indicated times and collected for protein extraction. Western blots were conducted to measure the levels of pTau S404, pTau S422, pTauS396, Tau, WWOX, cyclin D1, phospho-GSK3 $\beta$ S9, GSK3 $\beta, \beta$-catenin, phospho- $\beta$-catenin and actin. (c) Western blot results of Figure $2 \mathrm{~b}$ were quantified by scanning the autoradiogram using a densitometer. (d) SH-SY5Y cells were transfected with two pre-designed siRNA targeting WWOX for $24 \mathrm{~h}$, and the resulting cell lysates were subjected to western blot analysis. (e) SH-SY5Y cells were transfected with either control siRNA or WWOX siRNA as indicated. After $24 \mathrm{~h}$ of transfection, cells were washed, and the medium was replaced with $1 \%$ serum including $10 \mu \mathrm{M}$ RA. Cells with neurites longer than twice the length of the cell body were counted. Data are presented as the mean \pm S.E. from three independent experiments. ${ }^{*} P<0.05$, ${ }^{\star \star} P<0.01$ when compared with the mock control 
was observed in the RA-treated SH-SY5Y cells, accompanying the decreased phosphorylation of Tau at both S396 and S404; phosphorylation at S422 was unchanged (Figures $1 \mathrm{~b}$ and $\mathrm{c}$ ). Since studies have shown that S404 and S396 may be phosphorylated by GSK3 $\beta$ in vivo, ${ }^{16}$ we also examined whether the phosphorylation level of GSK3 $\beta$ and its downstream target, $\beta$-catenin, are affected by RA treatment. We found that the phosphorylation levels of phospho-GSK3 $\beta$ S9 and phospho- $\beta$-catenin remained normal. We therefore suspected that the reduced GSK3 $\beta$ mediated Tau phosphorylation and increased WWOX expression are indispensable for RA-induced SH-SY5Y cell differentiation. Furthermore, SH-SY5Y cells in which WWOX expression was reduced by RNAi showed increased pTau S396 levels and notably decreased neurite outgrowth (Figures 1d and e). Collectively, these results indicate that WWOX may have a vital role in SH-SY5Y cell differentiation and in Tau phosphorylation.
WWOX interacts and colocalises with GSK3 $\beta$ in vivo. Hernández et al. ${ }^{16}$ demonstrated that S396 and S404 on Tau are GSK3 $\beta$-dependent phosphorylation sites. Thus, we presumed that WWOX-dependent dephosphorylation of Tau occurred through an inhibition of GSK3 $\beta$ activity. We performed a bioinformatic analysis to align WWOX with well-known GSK3 $\beta$ inhibitors (Figure 2a) and found that WWOX $_{296-320}$ and WWOX $_{388-412}$ contain FXXXLI/ VXRLE, a highly conserved GSK3 $\beta$-binding motif within GSKIP $_{115-139}$, Axin $381-405$, and FRAT $205-229$. Previous reports have shown that $\mathrm{GID}_{381-405}$, a 25-amino acid region of Axin, is critical for its association with GSK $3 \beta .^{8}$ Interestingly, WWOX $296-320$ and WWOX $_{388-412}$ are similar to GID $_{381-405}$ in Axin (Figure 2a). WWOX W $_{296-320}$ is $40 \%$ similar to Axin $1_{381-405}, 32 \%$ similar to Axin2 $363-387,36 \%$ similar to FRAT $1_{205-229}$ and $34 \%$ similar to GSKIP $_{115-139}$. WWOX $_{388-412}$ is $26 \%$ similar to Axin $1_{381-405}, 34 \%$ similar to Axin2 $363-387,36 \%$ similar to FRAT1 $1_{205-229}$ and $32 \%$

a WWOX: 296SKLCNIIESNEZHRRLSPRGVTSNA 320

AXin1: 381 VRVEPQKEAEELIHRLEAVQRTREA $40540 \%$

Axin2: 363 TPVEPAT FAAELISELEKLKLELES $38732 \%$

FRAT1: 205 QLVLSGNLI KEAVRRLHSRRLOLRA $22936 \%$

GSKIP: 115 SPAYREA EGNALLCRLEALKRDGQS $13934 \%-$

WWOX: 388QSEETARTLWAISERIIQERLGSQS 412

Axinl: 381 VRVËPQKEAEELIHRLEAVŌRTREA $40526 \%$

Axin2: 363 TPVEPAT

FRAT1: 205 QLVLSGNLIKEAVRRLHSRRLQLRA 229 36\% -

GSKIP: 115 SPAYREA GGNALLEREALKRDGQS $13932 \%$
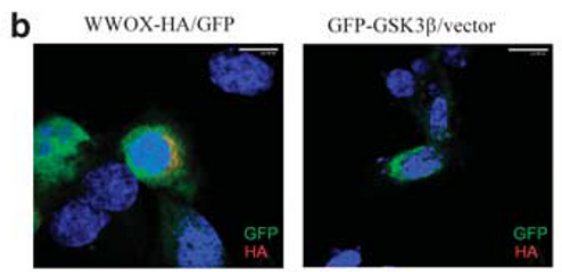

WWOX-HA/GFP-GSK3P

C

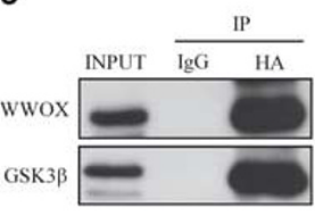

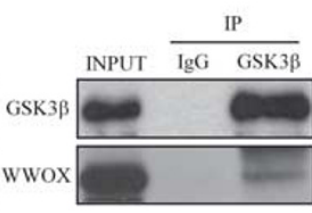

d

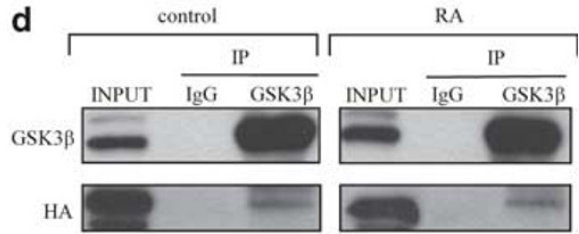

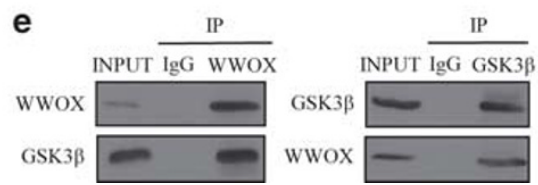

Figure 2 WWOX physically interacts and colocalises with GSK3 $\beta$ in vitro and in vivo. (a) Upper panel: the amino acid sequence of WWOX $296-320$, which is similar to highly conserved regions of Axin1 $_{381-405}$, Axin2 $_{363-387}$, FRAT1 $_{205-229}$ and GSKIP ${ }_{115-139}$, is highlighted in grey. Lower panel: the amino acid sequence of WWOX $388-412$, which is similar to highly conserved regions of Axin1 ${ }_{381-405}$, Axin2 $2_{363-387}$, FRAT1 $_{205-229}$ and GSKIP ${ }_{115-139}$, is highlighted in grey. Identical amino acids are highlighted in black. (b) SH-SY5Y cells were co-transfected with pEGFP-GSK3 $\beta$ and pcDNA3.1-HA-WWOX constructs for $24 \mathrm{~h}$. Cells were fixed with $3.7 \%$ formaldehyde, permeabilised, and incubated with mouse anti-HA primary antibodies and rhodamine-conjugated secondary antibodies. The cells were then observed using a Zeiss LSM510 confocal microscope. (c) Cells were harvested for immunoprecipitation using anti-WWOX (left) or anti-GSK3 $\beta$ (right) antibodies. WWOX-associated GSK3 $\beta$ was recognised by antiGSK3 $\beta$. Anti-HA was used to detect overexpressed HA-tagged WWOX. (d) SH-SY5Y cells were transiently transfected with the WWOX-HA construct and treated with RA for $24 \mathrm{~h}$. The cells were harvested for immunoprecipitation with anti-GSK3 $\beta$. Immunoprecipitated GSK3 $\beta$ was recognised by antibodies against GSK3 $\beta$. Anti-HA was used to detect HA-tagged WWOX. (e) Mouse brain extract was subjected to immunoprecipitation using anti-WWOX or anti-GSK3 $\beta$ as indicated. The immunoprecipitated complex was separated by molecular weight using SDS-PAGE. Anti-WWOX or anti-GSK3 $\beta$ antibodies were used to detect WWOX or GSK3 $\beta$ on the PVDF membrane 
similar to GSKIP $115-139$ (Figure 2a). These results suggested that WWOX may interact with GSK3 $\beta$. Hence, we performed immunofluorescence and immunoprecipitation experiments to determine whether WWOX is associated with GSK3 $\beta$. First, SH-SY5Y cells were co-transfected with plasmids expressing GSK3 $\beta$ fused to green fluorescent protein (GFP) (GFP-GSK3 $\beta$ ) and WWOX-HA to examine the in vivo association of WWOX and GSK3 $\beta$. Confocal fluorescent microscopy demonstrated that WWOX and GSK3 $\beta$ colocalised in the cytoplasm of SH-SY5Y cells (Figure 2b). We then performed co-immunoprecipitation experiments to confirm the in vivo interaction between WWOX and GSK3 $\beta$ in SH-SY5Y cells. Cell extracts were prepared from SH-SY5Y cells that had been transiently transfected with the WWOX-HA construct. Figure 2c (left panel) shows that the anti-HA antibodies precipitated HA-tagged WWOX, and that endogenous GSK3 $\beta$ coprecipitated with the WWOX protein complex. Similarly, ectopic HA-tagged WWOX was co-immunoprecipitated by anti-GSK3 $\beta$ antibodies (Figure 2c, right panel).

We considered the possibility that WWOX could regulate Tau phosphorylation through direct interaction. In Supplementary Figure A, however, we show that although WWOX$\mathrm{HA}$ can be immunoprecipitated with anti-HA antibodies, Tau was not co-immunoprecipitated in the complex (Supplementary Figure A), indicating that WWOX does not stably interact with Tau in SH-SY5Y cells. To clarify whether the interaction between WWOX and GSK3 $\beta$ is affected by RA treatment, we performed co-immunoprecipitation experiments in cells that had been stimulated with RA. SH-SY5Y cells were transiently transfected with the WWOX construct to mimic the upregulation of WWOX by RA treatment. Figure $2 d$ shows that the amount of GSK3 $\beta$ co-immunoprecipitated by WWOX from RA-treated SH-SY5Y cells was similar to the amount precipitated in untreated controls. Subsequently, immunoprecipitation was performed in mouse brain extracts to verify the physiological interaction between WWOX and GSK3 $\beta$. Figure $2 \mathrm{e}$ shows that both GSK3 $\beta$ and WWOX were precipitated by anti-GSK3 $\beta$ or anti-WWOX antibodies. These results indicate that WWOX physiologically associates and colocalises with GSK3 $\beta$ in vivo.

The WWOX-GSK3B interaction is mediated by a conserved motif in WWOX. To narrow down the binding site on WWOX that is responsible for its binding to GSK3 $\beta$, various WWOX functional domains, ww1 (1-60a.a.), ww2 (40-110a.a.), ww (1-110a.a.) and ADH (110-414a.a.), were expressed as glutathione $S$-transferase (GST) fusions in $E$. coli and affinity-purified on glutathione beads (Figure 3a). As shown in Figure 3b, the result of GST pulldown assay reveals an intense anti-GSK3 $\beta$ band was bound by the full-length WWOX and ADH-domain affinity matrix (Figure 3b, lanes 6 and 7), but not by GST alone (lane 2) or the other WWOX domains (lanes 3-5) examined. These results suggest that GSK $3 \beta$ interacts with WWOX by binding to its $A D H$ domain.

Additionally, the GST pull-down assay demonstrated that neither WWOX- $\Delta 389$ (1-389 a.a.) nor WWOX- $\Delta 286$ (1-286a.a.) were able to interact with GSK3 $\beta$ (Figure 3c). This indicates that WWOX amino acids 388-407 are required for its interaction with GSK3 $\beta$. Furthermore, bioinformatics analyses revealed that $L 404$ was highly conserved in WWOX and other GSK3 $\beta$-binding proteins (Figure $2 \mathrm{a}$ ). In order to clarify whether L404 within the highly conserved GSK3 $\beta$ binding motif, LXXRL, has an important role in GSK3 $\beta$ binding, a GST pull-down assay was performed using two WWOX mutants, L404A and L311A. As shown in Figure 3d, the mutation of L404A, but not L311A, completely abolishes the binding of WWOX to GSK3 $\beta$. These results suggest that WWOX physically interacts with GSK3 $\beta$ in vitro through the L404-containing motif within the WWOX $_{388-407}$ region. Finally, the co-immunoprecipitation experiment was performed again to compare wild-type (WT) WWOX with these mutants. As expected, ectopic GFP-WWOX can be co-immunoprecipitated by endogenous GSK3 $\beta$, and this interaction was diminished when L404 mutated to alanine (Figure $3 e$, right panel). Similar results were found in a co-immunoprecipitation experiment using GFP antibodies against GFP-tagged WWOX proteins (Figure 3e, left panel).

To investigate the possibility that the L404 mutant is incorrectly folded, thus disrupting GSK $3 \beta-$ WWOX interaction, we conducted co-immunoprecipitation experiments using c-jun, a WWOX-binding protein. C-jun was immunoprecipitated by either WT or mutant WWOX-GFP, indicating that WWOX L404A is folded correctly. ${ }^{17}$ To understand how WWOX associates with GSK3 $\beta$, we simulated the interaction in silico using molecular modelling. Figure $3 f$ illustrates the

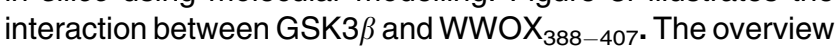
in Figure $3 f$ shows that the motif portion of WWOX $_{388-407}$ (highlighted in light pink and dark pink, respectively), especially L400, R403 and L404, are in close contact with a binding pocket $\alpha$-helix (G262-L273, red) and loop (N285$\mathrm{H} 299$, green) on GSK3 $\beta$. Figures $3 \mathrm{~g}$ and $\mathrm{h}$ and Supplementary Figure B show close-up views of WWOX $_{388-407}$ interacting with the binding pocket $\alpha$-helix and loop, respectively. As indicated in Figure $3 \mathrm{~g}$, the interactions between WWOX $_{388-407}$ and the binding pocket $\alpha$-helix are hydrophobic (indicated by red dashed lines) and electrostatic (indicated by green dashed lines). The van der Waals interactions included WWOX $_{388-407}$ L400 with a number of consecutive hydrophobic residues, including V263, L266, V267 and I270; WWOX $_{388-407}$ L404 with V267 and L270; and WWOX $388-407$ A399 with V263. A similar helix-helix ridge-groove hydrophobic interaction was also present in the GSK3 $\beta$-GSKIPtide $^{18}$ and GSK3 $\beta$-AxinGID ${ }^{19}$ structures. Similarly, the contact between WWOX $_{388-407}$ and the GSK3 $\beta$-binding loop was a simple hydrophobic interaction between $L 400$ and F293, and between L404 and I296. Collectively, these results revealed that the L404-containing motif located within WWOX $_{388-407}$ is essential for the association of WWOX and GSK3 $\beta$.

GSK3 $\beta$ kinase activity is negatively regulated by WWOX. We next investigated the functional effect of WWOX in regulating GSK3 $\beta$ activity. We performed an in vitro kinase assay to examine whether WWOX interferes with GSK3 $\beta$-mediated Tau phosphorylation. GST-tagged WWOX protein $(0.3 \mu \mathrm{g})$ and His-GSK3 $\beta$ protein (30 ng) were incubated in reaction buffer for $30 \mathrm{~min}$ before adding $0.5 \mu \mathrm{g}$ His-Tau protein and a $\left[\gamma^{32} \mathrm{P}\right]$ ATP cocktail. 
a

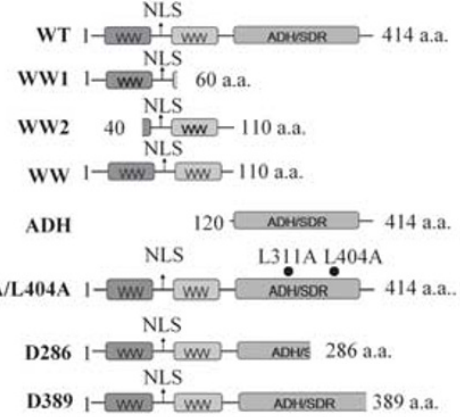

b
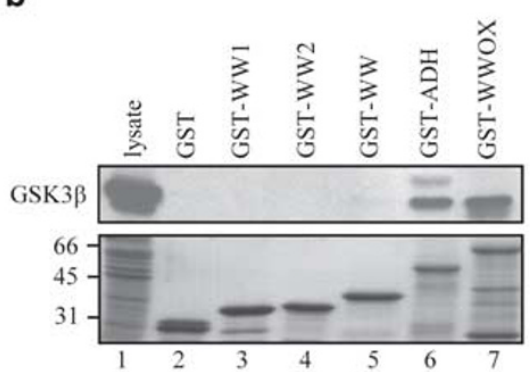

C

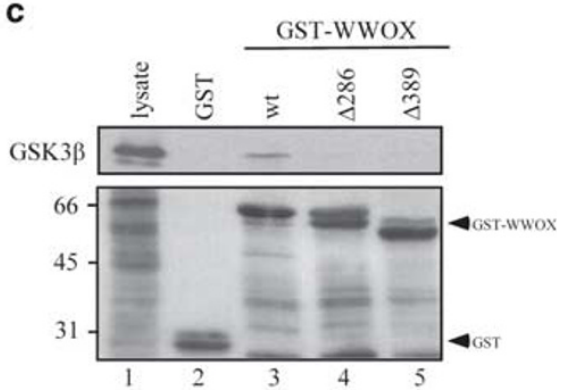

d

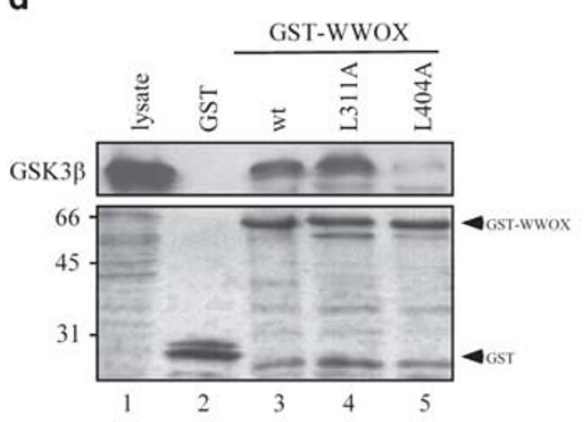

e

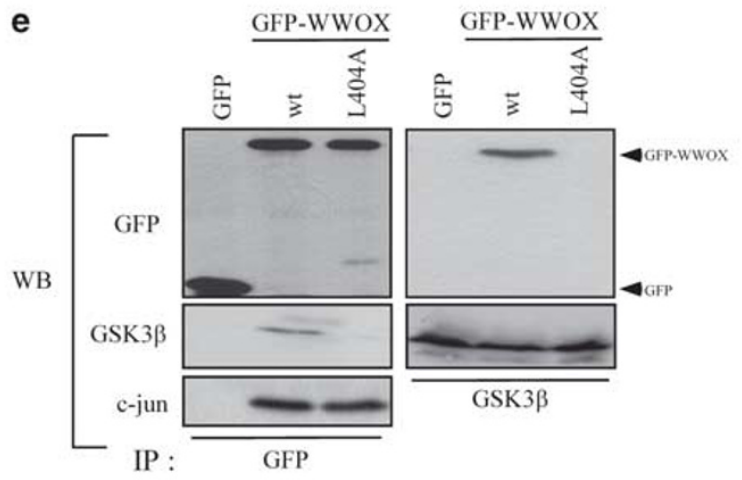

f

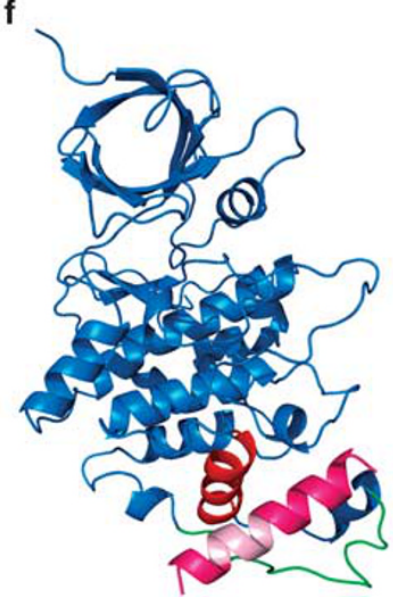

g

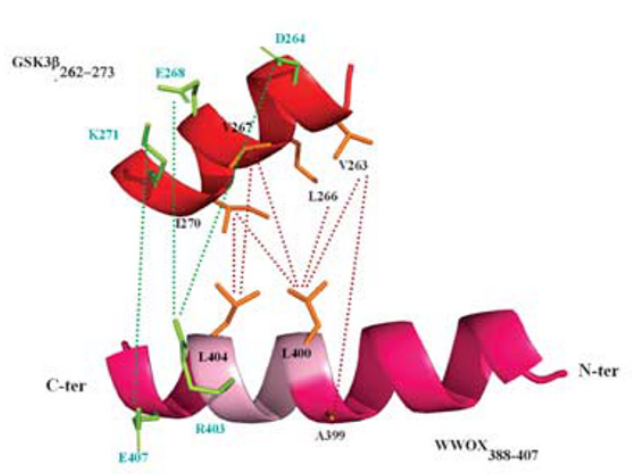

h

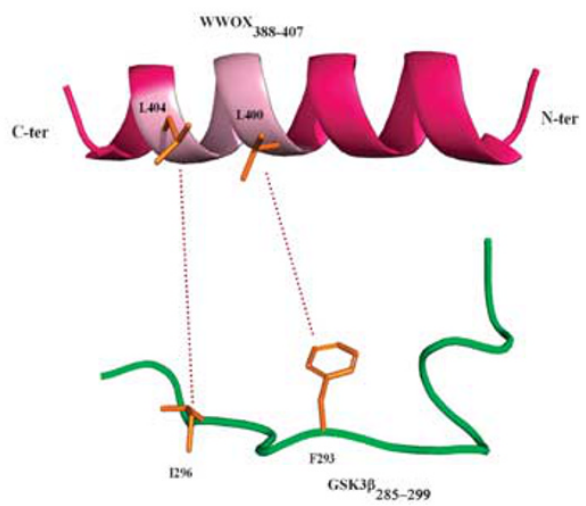

Figure 3 Leucine 404 within the ADH domain is critical for WWOX associated with GSK3 $\beta$. (a) WWOX constructs used in this study. Full-length WwOX, ww1, ww2, ww, ADH domain and mutant WWOX (L311A and L404A) were cloned into a GST-fusion protein expression vector. Two partial deletions of the WWOX ADH domain are indicated ( $\Delta 389$ and $\Delta 286$ ). (b) Each GST-tagged WWOX construct was prepared and incubated with cell lysates in a binding buffer. Equivalent loading of the proteins was confirmed using Coomassie blue staining. Western blot analysis with anti-GSK3 $\beta$ antibodies was used to verify the interaction domain. (c) GST, WWOX and two WWOX-derived GSTfusion proteins ( $\Delta 389$ and $\Delta 286$ ) were analysed in a GST pull-down assay. (d) GST pull-down assay with GST or GST-fused mutant WWOX (L311A and L404A). (e) SHSY5Y cells were transfected with GFP, GFP-WWOX or GFP-WWOXL404A and harvested for immunoprecipitation with anti-GSK3 $\beta$. Anti-GFP was used to detect GFP-WWOX. (f-h) Molecular modelling of the WWOX-GSK3 $\beta$ interaction. (f) An overview of the interaction of WWOX B $_{38-407}$ and GSK3 $\beta$. (g) A close-up view of the

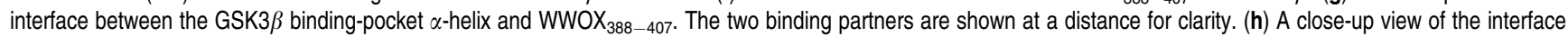
between the GSK3 $\beta$-binding pocket loop and WWOX W88-407. The two binding partners are shown at a distance for clarity. Colour code: WWOX $_{388-407}$, pink; GSK3 $\beta$-binding pocket $\alpha$-helix, red; GSK3 $\beta$-binding pocket loop, green; GSK3 $\beta$ non-interacting regions, blue. Red dashed lines indicate electrostatic interactions, namely hydrogen bonds or salt bridges; green dashed lines indicate hydrophobic interactions

The GSK3 $\beta$-mediated Tau phosphorylation was examined by autoradiography and western blotting analysis. As shown in Figure $4 \mathrm{a}, \mathrm{GSK} 3 \beta$ can phosphorylate Tau in vitro, and this phosphorylation is completely abolished in the presence of excess WWOX, but not GST or WWOX L404A. Moreover, we demonstrated that GSK3 $\beta$-mediated Tau phosphorylation at S396 and S404 was reduced in a dose-dependent manner by GST-WWOX (Figure 4b). In the same experiment, we investigated the specificity of the effect of WWOX on GSK3 $\beta$ mediated Tau phosphorylation by examining the phosphorylation of S422, which is phosphorylated by MKK4 kinase in vivo (Figure 4c). SH-SY5Y cells were transiently transfected with GFP and GFP-WWOX for $12 \mathrm{~h}$, and the cell lysate was subjected to western blotting. Ectopically 

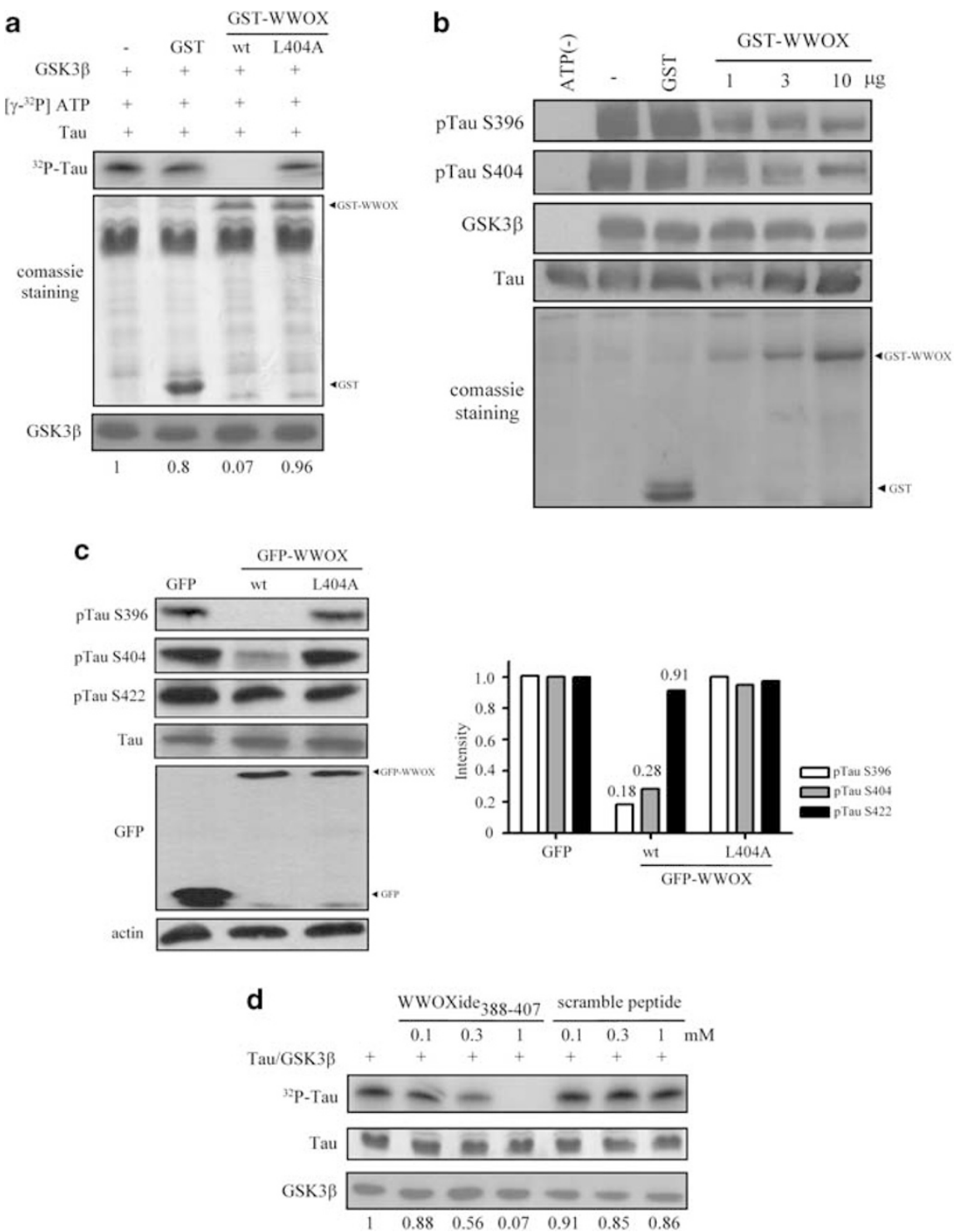

Figure 4 WWOX suppresses Tau phosphorylation. (a) GST, GST-WWOX or GST-WWOX L404A (0.2 $\mu \mathrm{g})$ were incubated with $30 \mathrm{ng}$ His-GSK3 $\beta$ for 30 min in a reaction buffer and incubated with $0.5 \mu \mathrm{g}$ Tau protein, ATP cocktail, and $10 \mathrm{Ci}$ of $\left[\gamma^{32} \mathrm{P}\right]$ ATP for $10 \mathrm{~min}$ at $20^{\circ} \mathrm{C}$. The reaction was stopped by adding $2 \times$ sample buffer and heating at $100^{\circ} \mathrm{C}$ for $10 \mathrm{~min}$. This was followed by SDS-PAGE, and phosphorylation signals were evaluated using autoradiography. (b) WWOX (1, 3 and $\left.10 \mu \mathrm{g}\right)$ inhibited Tau phosphorylation by GSK3 $\beta$ in a dose-dependent manner. GST-WWOX was pre-incubated with His-GSK3 $\beta$ for 30 min in a reaction buffer including optimised quantities of ATP and Tau protein. The reactions were then subjected to western blot analysis of Tau, pTauS396 and S404. (c) SH-SY5Y cells were transiently transfected with GFP, GFP-WWOX or GFP-WWOX L404A. Cells were lysed, and a western blot analysis was carried out using antibodies against pTauS396, pTauS404, pTauS422, Tau and actin. Actin expression was evaluated to verify equal loading, and GFP expression was evaluated to confirm the protein expression level. (d) A scrambled peptide and WWOXtide $_{388-407}$ were each incubated with $30 \mathrm{ng}$ His-GSK3 $\beta$, ATP-cocktail, and $10 \mathrm{Ci}$ of $\left[\gamma^{32} \mathrm{P}\right]$ ATP for $10 \mathrm{~min}$ at $20^{\circ} \mathrm{C}$. The reaction was stopped by adding $2 \times$ sample buffer and was followed by SDS-PAGE. Phosphorylation was detected with autoradiography

expressed WWOX significantly inhibited Tau phosphorylation at S404 and S396 but not S422. However, this inhibition was diminished when WWOX L404A was used. These results again confirm our finding that the binding of WWOX to GSK3 $\beta$ is required for its inhibition of GSK3 $\beta$-mediated Tau phosphorylation. The phosphorylation of Tau S422 is not affected by WWOX (Figure 4c), suggesting that the inhibition of GSK3 $\beta$ through the binding of WWOX is highly specific, at least in SH-SY5Y cells. Moreover, we examined the effect of WWOX on the activity of GSK3 $\beta$ toward the peptide substrate GS-1, which is derived from glycogen synthase. Consistent with our observations of Tau phosphorylation, WT but not mutant WWOX inhibits GSK3 $\beta$ phosphorylation of GS-1 (Supplementary Figure C).

We subsequently performed a competitive inhibition assay to further support our conclusion using WWOXtide ${ }_{388-407}$, a WWOX-derived peptide, in the in vitro kinase assay experiment. WWOXtide $388-407$, but not the control scrambled peptide, inhibited GSK3 $\beta$ activity and reduced Tau phosphorylation, as shown by autoradiography (Figure 4d). Collectively, 
our results reveal that WWOX negatively regulates GSK3 $\beta$ kinase activity on the neuron-specific microtubule-binding protein Tau in SH-SY5Y cells.

WWOX promotes neuronal cell differentiation through the inhibition of GSK3 $\beta$. Researchers have suggested that GSK3 $\beta$ has an important role in cell differentiation. We therefore investigated whether GSK3 $\beta$ inactivation is involved in RA-stimulated neuronal cell differentiation. SH-SY5Y cells were transiently transfected with a GFP control or GSK3 $\beta$ (WT, S9A or kinase dead (KD), or R96A) for $24 \mathrm{~h}$, and the neurite outgrowth of SH-SY5Y cells, indicating neuronal cell differentiation, was analysed. Transfection with GFP-GSK3 $\beta$ WT and S9A notably decreased SH-SY5Y cell differentiation, whereas KD and R96A did not affect SH-SY5Y cell differentiation compared with the GFP control (Figures $5 a$ and $b$ ). GSK3 $\beta$ small interference RNA (siRNA) was used to knock down GSK3 $\beta$ protein expression to confirm the crucial role of GSK3 $\beta$ inactivation in RA-mediated axon growth. SH-SY5Y cells were transfected with GSK3 $\beta$ siRNA, followed by treatment with $10 \mu \mathrm{M}$ RA for 1 to 4 days. Compared with the control scrambled-RNAi group, the GSK3 $\beta$ protein-knockdown group showed significantly increased neurite outgrowth percentage beginning on the second day of RA treatment (Figures $5 c$ and $d$ ). Thus, our results showed that GSK3 $\beta$ could negatively regulate neurite outgrowth in RA-stimulated neuronal cells.

Microtubule polymerisation is indispensable in neurons because neurite growth is severely disrupted when polymerisation is inhibited, indicating that a constant flux of microtubules is required for axonal growth. Furthermore, evidence shows that the phosphorylation of Tau by GSK3 $\beta$ affects the ability of Tau to promote microtubule formation. ${ }^{20-22}$ This evidence, in addition to the results of this study, suggests that WWOX may promote neuronal cell differentiation by inhibiting GSK3 $\beta$-mediated Tau phosphorylation. We therefore used a light-scattering assay to clarify how WWOX and GSK3 $\beta$ affect Tau-mediated microtubule assembly. As shown in Figure 5e, we measured the effect of GSK3 $\beta$ on the turbidimetric time course of microtubule assembly. GSK3 $\beta$ diminished the microtubule assembly, as reflected by decreased turbidity. However, the new equilibrium reached a higher turbidity when treated with WT WWOX and GSK3 $\beta$ than with GSK3 $\beta$ alone. Interestingly, the GSK3 $\beta$-binding deficient WWOX, WWOX L404A, could not restore the microtubule assembly activity. In conclusion, our data demonstrated that WWOX could restore the ability of Tau to promote microtubule formation by inhibiting GSK3 $\beta$ activity.

WWOX L404A, which is deficient in binding to GSK3 $\beta$ (Figure 3 ) and was incapable of restoring Tau activity on microtubule formation (Figure $5 \mathrm{e}$ ), should fail to stimulate neuronal cell differentiation. To confirm this prediction, the SH-SY5Y cell differentiation promoted by WWOX L404A was examined and compared with that of WT WWOX. WT WWOX and WWOX L404A were separately overexpressed in SH-SY5Y in the presence of RA treatment. WWOX significantly increased the percentage of differentiated SH-SY5Y cells. However, this increase was abolished when L404 was mutated to alanine. These results strongly suggest that WWOX promotes neuronal differentiation in SH-SY5Y cells by inhibiting the GSK3 $\beta$-mediated phosphorylation of the microtubule-binding protein Tau (Figures $5 f$ and $g$ ).

To demonstrate that Tau is indeed the ultimate effector of WWOX/GSK3 $\beta$-dependent neuronal cell differentiation, we performed double manipulation of WWOX and GSK3 $\beta$, as well as the delivering of siRNA to knock down Tau and GSK3 $\beta$, respectively, and then conducted RA-induced neurite outgrowth experiments. Figures $6 a$ and b (lanes 1-3) show that the neurite outgrowth stimulated by RA was abolished when Tau was knocked down. Western blotting (Figure 6b) confirmed protein overexpression or knockdown after transient transfection of the WWOX-expressing vector or Tau and GSK3 $\beta$ siRNAs. Neuronal differentiation increased to $44 \%$ in the WWOX overexpression group comparing with $18 \%$ of RAcontrol cells group (lane 6). This increase was completely abolished when Tau was knocked down in a double manipulation experiment (lane 5). Similar results were exhibited in a GSK3 $\beta$ and Tau double manipulation experiment, which showed that neuronal cell differentiation of the GSK3 $\beta$ knockdown group increased approximately $25 \%$ more than the RA-control (lane 7), which was completely abolished when Tau was knocked down (lane 4). Neither WWOX overexpression nor GSK3 $\beta$ knockdown promoted neurite outgrowth in the Tau knockdown condition, indicating that Tau is the effector of both WWOX and GSK3 $\beta$ (Figures 6a and b). Interestingly, co-transfection of the WWOX plasmid and GSK3 $\beta$ siRNA followed by RA treatment did not show an additive or synergistic effect compared with WWOX overexpression alone or GSK3 $\beta$ knockdown alone (Figures 6a and $b$, lane 8). WWOX did not further promote neurite outgrowth in the presence of GSK3 $\beta$ siRNA, consistent with the hypothesis that WWOX promotes neuronal differentiation through GSK3 $\beta$ inhibition. Collectively, these results connect WWOX, GSK3 $\beta$ and Tau in an exclusive, direct manner and show that WWOX promotes neuronal SH-SY5Y cell differentiation through inhibiting the GSK3 $\beta$-mediated phosphorylation of the microtubule-binding protein, Tau.

\section{Discussion}

In this study, we found two GSK3 $\beta$ consensus-binding sites in the WWOX amino-acid sequence. Furthermore, our results show that WWOX physically interacts with GSK3 $\beta$ only through the WWOX $_{388-407}$ region, which is an L404-containing motif. Some reports suggest that $L 400$ is also important for WWOX to associate with GSK3 $\beta$. Smalley et al. ${ }^{19}$ demonstrated that L392 and L396 are essential for the interaction between Axin1 and GSK3 $\beta$. Interestingly, the L392 and L396 of Axin1 are related to $L 400$ and $L 404$, respectively. These results show that WWOX interacts with GSK3 $\beta$ through a structure and sequence similar to that of Axin $_{381-405}$. Furthermore, researchers have suggested that GSK3 $\beta$ has an important role in multiple cellular processes, including protein synthesis, glycogen metabolism, proliferation, microtubule dynamics and cell differentiation. ${ }^{23}$ WWOX may help to control certain types of cellular processes through the inhibition of GSK3 $\beta$.

WWOX expression in the developing brain is low during the early embryonic stage when organogenesis is most rapid; however, moderate-to-high WWOX immunoreactivity is 


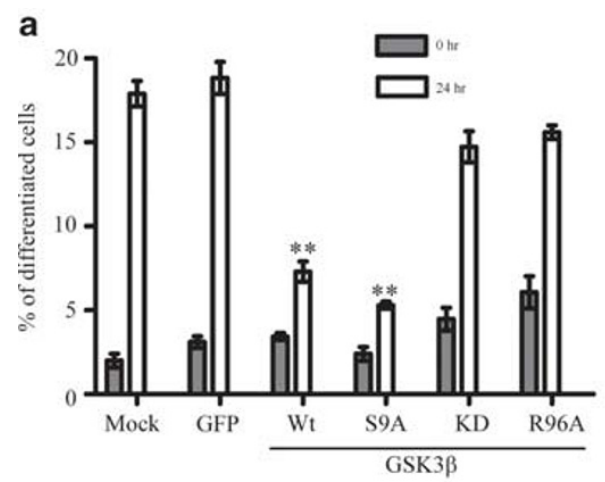

b

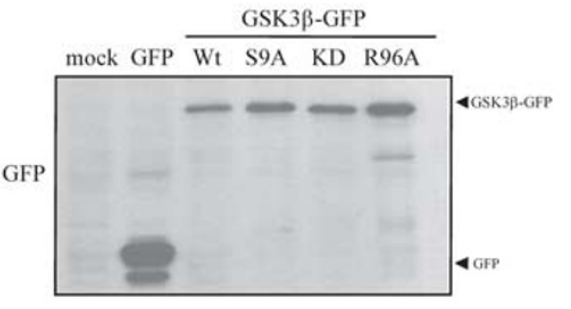

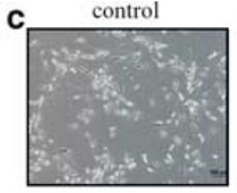
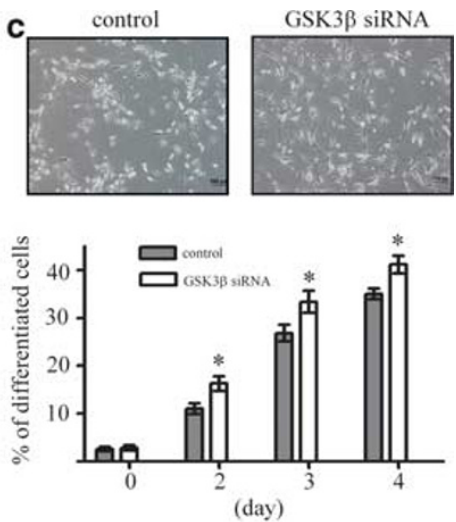

d

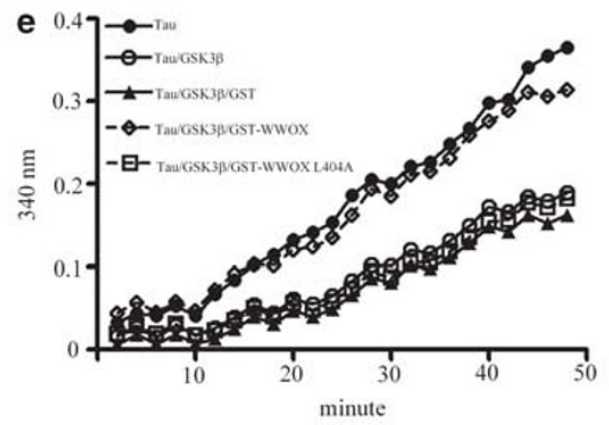

f

GFP
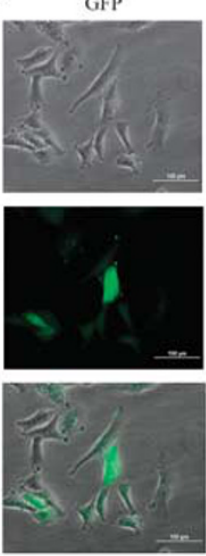

WWOX-GFP
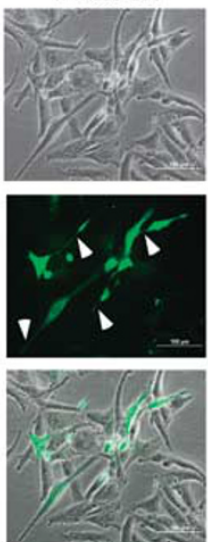

WWOX-GFP L 404A
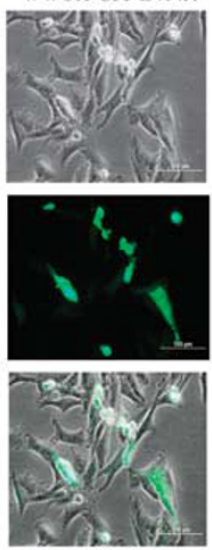

g
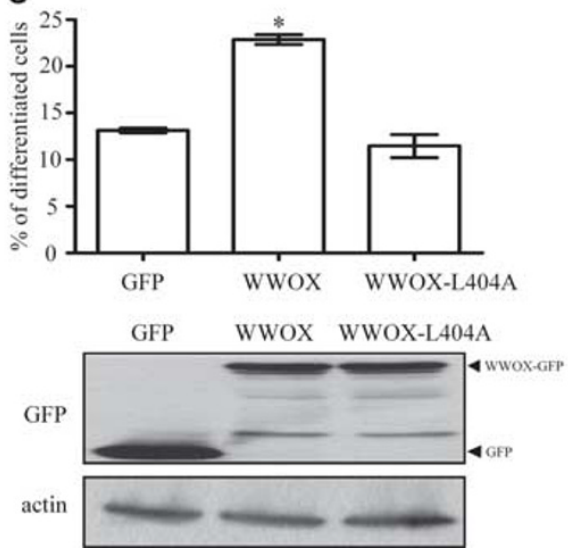

Figure 5 WWOX regulates neuronal differentiation through its interaction with GSK3 $\beta$. (a) SH-SY5Y cells were transfected with GFP, GFP-GSK3 $\beta$ WT, S9A, K8586Ml and R96A for $24 \mathrm{~h}$. The cells were then treated with $10 \mu \mathrm{M}$ RA for $24 \mathrm{~h}$. Cells with neurites longer than twice the length of the cell body were counted. Data are presented as the mean \pm S.E. from three independent experiments. ${ }^{*} P<0.01$ compared with the GFP control. (b) Western blot analysis confirmed the expression of the GFP-fusion protein by the anti-GFP antibody. (c) SH-SY5Y cells were transfected with pre-designed siRNA targeting GSK3 $\beta$ for $24 \mathrm{~h}$ and treated with $10 \mu \mathrm{M}$ RA for 1 to 4 days. Cells with neurites longer than twice the length of the cell body were counted. Bright-field results are shown to indicate post-differentiation status at $24 \mathrm{~h}$. Data are presented as the mean \pm S.E. from three independent experiments. ${ }^{*} P<0.05$ compared with mock control. (d) Cells were treated with siRNA and lysed in $2 \times$ sample buffer. Western blot analysis was carried out using antibodies against GSK3 $\beta$. The blot was stained for actin to verify equal loading. (e) Tau protein was incubated with GST, GST-WWOX or GST-WWOX L404A in the presence of GSK3 $\beta$ for the microtubule assembly assay. The reaction mixture also contained tubulin protein, GTP, and reaction buffer. The reaction mixture was incubated at $37^{\circ} \mathrm{C}$ in a thermostatic spectrophotometer, and the change in reflectance over time was measured at $350 \mathrm{~nm}$. (f) $\mathrm{SH}-\mathrm{SY} 5 \mathrm{Y}$ cells were transfected with empty vector, GFP-WWOX or GFP-WWOXL404A, as indicated. After transfection, cells were washed, and the medium was replaced with $1 \%$ serum and treated with $10 \mu \mathrm{M}$ RA. The photos were taken on day 1. (g) The percentage of differentiated cells with neurites longer than twice the length of the cell body was calculated. The data are presented as the mean \pm S.E. from three independent experiments. ${ }^{*} P<0.05$ compared with the mock-treated control

detected in the brain and spinal cord during the middle and late fetal stages. ${ }^{12}$ This strongly suggests that WWOX may have an important role in neuron differentiation. In this study, highly differentiated SH-SY5Y cells were correlated with high WWOX protein expression and low Tau S396 and S404 protein phosphorylation. This indicates that SH-SY5Y cell differentiation requires WWOX protein expression and lower pTau S396 and pTau S404 levels, possibly caused by the inhibition of GSK3 $\beta$. Intriguingly, Sze's group demonstrated that the downregulation of WWOX induces 
a

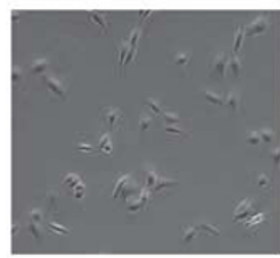

RA/Tau siRNA/pcDNA3 -WWOX

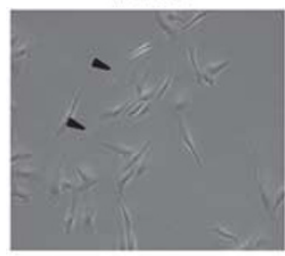

b

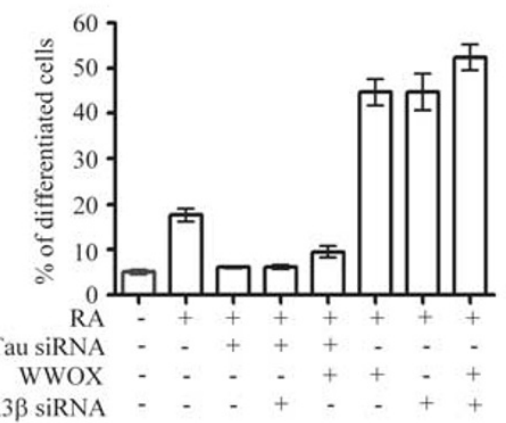

RA/scramble siRNA

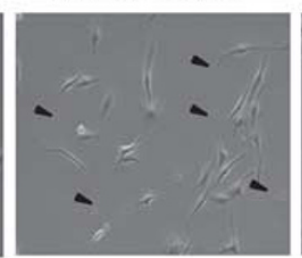

RA/pcDNA3-WWOX

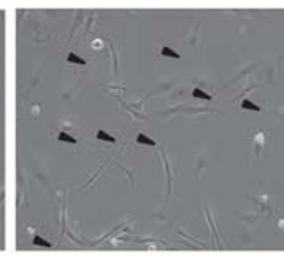

C
RA/Tau siRNA/GSK3 3

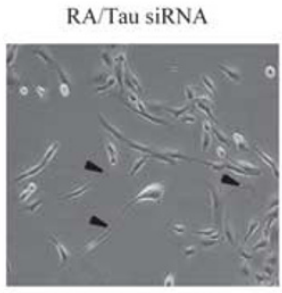

RA/GSK3ß siRNA

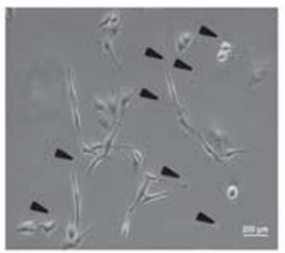

RA/GSK $3 \beta$ SIRNA/WWOX siRNA
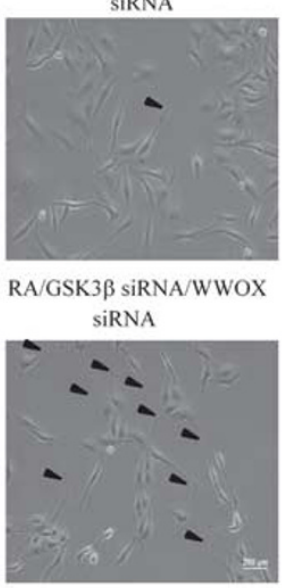

C
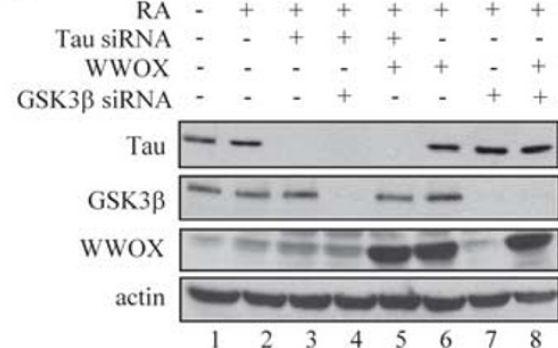

d

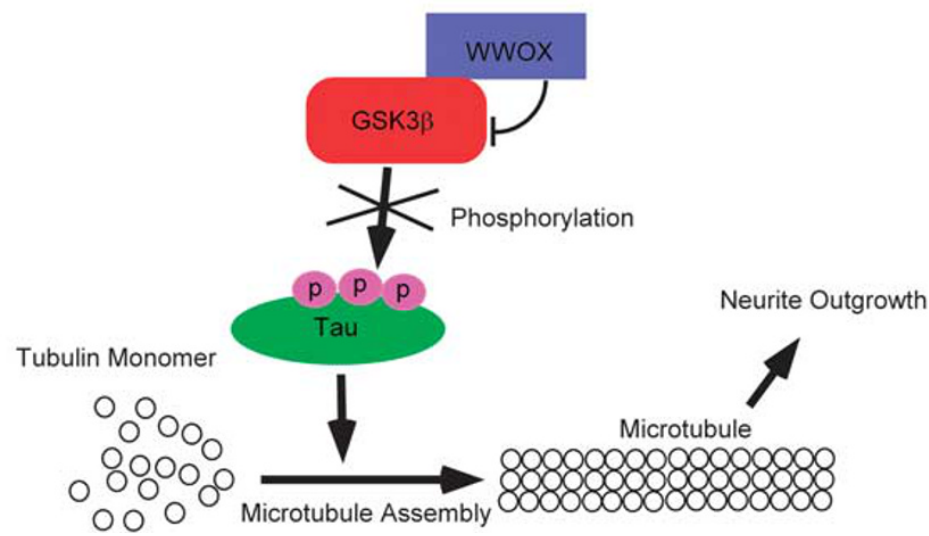

Figure 6 Tau is indispensable for WWOX regulation of neuronal differentiation. (a) SH-SY5Y cells were transfected with Tau siRNA, GSK3 $\beta$ siRNA and WWOX together or separately for $24 \mathrm{~h}$. They were then treated with $10 \mu \mathrm{M}$ RA for another $24 \mathrm{~h}$. The photos were taken on day 1 . (b) The percentage of differentiated cells with neurites longer than twice the length of the cell body was calculated. (c) Cells treated with Tau and GSK3 $\beta$ siRNA and WWOX plasmid were lysed in $2 \times$ sample buffer, and a western blot analysis was carried out using antibodies against Tau, WWOX and GSK3 $\beta$. (d) Our proposed model, showing that WWOX abolishes GSK3 $\beta$ kinase activity and therefore restores the ability of Tau to promote microtubule assembly

Tau phosphorylation. They also showed that WWOX was downregulated in $A D$ patients. ${ }^{2}$ Furthermore, the level of active GSK3 $\beta$ is reportedly increased in AD. ${ }^{24}$ Based on our results, we speculate that the upregulation of GSK3 $\beta$, and therefore Tau phosphorylation, in AD may due to the downregulation of WWOX.

Chen et al. ${ }^{12}$ reported that WWOX was differentially expressed during various stages of brain development in mice, indicating its potential role in promoting neuronal differentiation and maturation. They also used a yeast two-hybrid assay to demonstrate that WWOX associates with Tau and showed that the downregulation of WWOX may induce Tau phosphorylation in vitro. ${ }^{2}$ However, the molecular mechanism by which WWOX facilitates neural differentiation has yet to be revealed. Here, we were not able to detect a Tau-WWOX complex in our co-immunoprecipitation experiment from SH-SY5Y cells (Supplementary Figure A); the inconsistency in results may due to the different expression systems used. However, our results connect WWOX, GSK3 $\beta$ and Tau in an exclusive, direct manner and describe a novel 
mechanism by which WWOX promotes neuronal SH-SY5Y cell differentiation by inhibiting the GSK3 $\beta$-mediated phosphorylation of the microtubule-binding protein, Tau.

The extension of immature neurites requires the assembly of a stable microtubule infrastructure. Microtubule polymerisation and stability are regulated by a number of microtubuleassociated proteins, including Tau. GSK3 $\beta$ regulates Tau through phosphorylation; hyperphosphorylated Tau has a decreased affinity for microtubules and disrupts microtubule stability. This study shows that the knockdown of GSK3 $\beta$ expression through RNAi decreases Tau phosphorylation and increases the occurrence of SH-SY5Y cell differentiation. Additionally, researchers have reported that the inactivation of GSK3 $\beta$ results in the dephosphorylation of the GSK3 $\beta$ downstream target, CRMP-2, which leads to enhanced microtubule polymerisation and axon growth. ${ }^{11}$ Therefore, neurite outgrowth requires microtubule assembly and the inhibition of GSK3 $\beta$ activity. However, a number of previous studies have reported that the activation of GSK3 $\beta$ enhanced neurite growth rates in PC12, NT2 or SH-SY5Y cells. ${ }^{25-27}$ However, our microtubule assembly assay clearly showed that WWOX restores the microtubule assembly-promoting activity of Tau that is repressed by GSK3 $\beta$. Ahmad et al. ${ }^{21}$ reported that the inhibition of microtubule polymerisation compromises axonal growth. We observed highly phosphorylated Tau in undifferentiated SH-SY5Y cells. Reduced Tau phosphorylation also correlated strongly with WWOX expression in highly differentiated SH-SY5Y cells. These results provide evidence that WWOX has an important role in inhibiting GSK3 $\beta$ activity and suppressing Tau hyperphosphorylation.

In conclusion, our study demonstrates that WWOX physically interacts with GSK3 $\beta$ through its ADH/SDR domain and decreases Tau protein hyperphosphorylation at S404 and S396, both in vivo and in vitro. Furthermore, we showed that an increased WWOX protein level is correlated with decreased pTau S396 and S404 levels in highly differentiated SH-SY5Y cells. The microtubule assembly will eventually be promoted by Tau when GSK3 $\beta$ is inhibited by WWOX during RA-stimulated neuronal cell differentiation. We thus present a novel mechanism for the regulation of neuronal differentiation by WWOX.

\begin{abstract}
Materials and Methods
Cell culture, transfection and treatments. Human neuroblastoma SH-SY5Y cells were maintained in Dulbecco's Modified Eagle's Medium supplemented with $10 \%$ fetal calf serum and antibiotics $(100 \mathrm{mg} / \mathrm{ml}$ streptomycin and $100 \mathrm{IU} / \mathrm{ml}$ penicillin; Life Technologies, Inc., Gaithersburg, MD, USA). The cells were grown to subconfluence and then treated with $10 \mu \mathrm{M}$ RA for 1 to 5 days. The information of siRNA used in this study was provided in Supplementary Table 1. For efficient transfection, Lipofectamine 2000 (Invitrogen, Carlsbad, CA, USA) was used according to the manufacturer's instructions.
\end{abstract}

Plasmid construction. A full-length WWOX (human WOX1) construct was subcloned into the following expression vectors: pEGFP-C1-CPO, pGEX-KG and pcDNA3.1-HA-CPO. The ADH domain fragment was generated by PCR with pfu polymerase (Promega, Madison, WI, USA). The PCR product was subcloned into the pGEX-KG, pEGFP-C1-CPO and pcDNA3.1-HA-CPO plasmids for sequence verification and further cloning.

WWOX mutants (L311A and L404A) were prepared using site-directed mutagenesis and a two-step PCR technique. After successful cloning, the WWOX CDNA insert sequences were further confirmed through DNA sequencing.
Western blotting. Cells were disrupted in $2 \mathrm{X}$ sample buffer $(0.1 \mathrm{M}$ Tris- $\mathrm{HCl}$ $\mathrm{pH} 6.8,4 \% \mathrm{SDS}, 20 \%$ glycerol, $2 \% \beta$-mercaptoethanol), boiled for $10 \mathrm{~min}$, centrifuged, placed on ice for several minutes, stored at $-80^{\circ} \mathrm{C}$ and then separated using $10-12.5 \%$ SDS-polyacrylamide gel electrophoresis (PAGE). Proteins were then electrotransferred onto PVDF transfer membranes (Millipore, Lincoln Park, NJ, USA). The membranes were blocked with $5 \%$ nonfat milk in TBST buffer $(10 \mathrm{mM}$ Tris- $\mathrm{HCl} \mathrm{pH} \mathrm{7.4,} 150 \mathrm{mM} \mathrm{NaCl}, 0.5 \%$ Tween 20) containing $5 \%$ bovine serum albumin at $4{ }^{\circ} \mathrm{C}$ for $2 \mathrm{~h}$ or overnight, and incubated for $2 \mathrm{~h}$ at room temperature with one of the following primary antibodies: monoclonal anti-GFP (Santa Cruz Biotechnology, Santa Cruz, CA, USA), anti-GSK3 $\beta$ (BD Transduction Laboratories, Lexington, KY, USA), monoclonal anti-actin (Sigma, St. Louis, MO, USA), Tau (Millipore), rabbit anti-pTau S396 (Origene, Rockville, MD, USA), rabbit anti-pTau S404 (Millipore) or rabbit anti-pTauS422 (Abcam, London, UK). The membranes were washed with TBST six times every $10 \mathrm{~min}$. Immunoreactive proteins were detected using horseradish peroxidase-conjugated secondary antibodies and then the membrane was washed with TBST six times every $10 \mathrm{~min}$ during the detection process. The protein signal was detected by an enhanced chemiluminescence system (ECL Plus; Perkin Elmer Life and Analytical Sciences, Inc., Waltham, MA, USA).

Immunoprecipitation and GST pull-down assays. For immunoprecipitation, SH-SY5Y cells were washed twice with phosphate-buffered saline (PBS) at the indicated times and lysed in ice-cold RIPA buffer (100 mM HEPES pH 7.4, $150 \mathrm{mM} \mathrm{NaCl}, 2 \mathrm{mM}$ EDTA, 0.5\% Tween 20, 0.1\% Triton X-100, $1 \mathrm{mM} \mathrm{DTT}, 50 \mu \mathrm{g} / \mathrm{ml}$ AEBSF, $10 \mu \mathrm{g} / \mathrm{ml}$ leupeptin, $10 \mu \mathrm{g} / \mathrm{ml}$ aprotinin, $5 \mathrm{mM} \mathrm{NaF}$ and $1 \mathrm{mM} \mathrm{Na}_{3} \mathrm{VO}_{4}$ ). Anti-WWOX antibodies were incubated with Protein G-Sepharose beads for $1 \mathrm{~h}$ at $4{ }^{\circ} \mathrm{C}$, and the pretreated cell lysates were added to the Protein G-Sepharose beads for $2 \mathrm{~h}$ at $4^{\circ} \mathrm{C}$. The immune complexes were then washed three times with a RIPA buffer, eluted by boiling in a 2X SDS sample buffer, and subjected to western blotting analysis. For GST pull-down assays, GST or GSTWWOX proteins expressed in BL21 (DE3) were adsorbed to glutathione-agarose beads (Sigma) for $1 \mathrm{~h}$ after three washes with PBS. The beads were then washed three times with the same buffer. Sample buffer was added, and the beads were boiled and subjected to western blot analysis.

Immunofluorescence assays. SH-SY5Y cells co-transfected with pcDNA3.1-HA-WWOX and GFP-GSK3 $\beta$ were grown on coverslips, washed in PBS, fixed with $3.7 \%$ paraformaldehyde in PBS, and permeabilised by incubation in $10 \%$ Triton X-100. Following permeabilisation, the cells were washed with PBS and incubated with a mouse anti-HA monoclonal antibody (Santa Cruz Biotechnology) at $4{ }^{\circ} \mathrm{C}$ overnight. The cells were washed again and then incubated with goat anti-mouse IgG rhodamine (Santa Cruz Biotechnology). The cells were washed again and subsequently stained with DAPI. In each instance, the cells were washed in PBS. Fluorescently labelled protein localisation was visualised using a Zeiss laser confocal microscope (LSM510; Zeiss Inc, Oberkochen, Germany).

In vitro kinase assay. His-tagged human Tau and His-tagged human GSK3 $\beta$ were expressed in $E$. coliBL21 and purified by means of affinity chromatography. The purity of the recombinant proteins was verified by SDS-PAGE. The purified recombinant GSK3 $\beta$ protein was pre-incubated with WT or mutant GST-WWOX and $25 \mu \mathrm{g} / \mathrm{ml} \mathrm{His-Tau} \mathrm{for} 30 \mathrm{~min}$, and the kinase reaction was initiated by the addition of an equal volume of $2 X$ reaction buffer (100 mM HEPES, pH 7.4, $1 \mathrm{mM} \mathrm{DTT}$ and $10 \%$ glycerol) and ATP cocktail (20 mM ATP and $1 \mathrm{mM} \mathrm{MgCl} 2$ ). After incubating the mixtures at $30^{\circ} \mathrm{C}$ for up to $20 \mathrm{~min}$, the reaction was stopped by adding $2 \times$ sample buffer and heating at $100^{\circ} \mathrm{C}$ for $10 \mathrm{~min}$. The proteins were further separated using SDS-PAGE. Results were detected using autoradiography or western blotting.

Molecular dynamics simulation. A molecular dynamics (MDs) simulation was employed to predict the binding interaction between GSK3 $\beta$ and WWOX $_{388-407}$. As the structure of WWOX is unknown, the GOR IV Secondary Structure Prediction Method ${ }^{28}$ was used to predict the structure of the WWOX $_{388-407}$ peptide. Accordingly, the structure of the GSK3 $\beta$-AxinGID complex (PDB entry code: 109U) was used as a template to create an initial structure for the GSK3 $\beta$-WWOX B $_{388-407}$ complex. The structure of AxinGID was extracted from the complex, and the amino acids were modified to generate WWOX $_{388-407}$. The resulting WWOX W $_{38-407}$ peptide was then subjected to energy minimisation in which the backbone was constrained and the side chains were allowed to achieve their proper orientations using 5000 steps of the steepest descent algorithm and 5000 steps of the conjugate gradient algorithm. The X-ray 
structure of GSK3 $\beta$-AxinGID served as a guide for orienting WWOX $\mathrm{X}_{388-407}$ in the GSK3 $\beta$-binding pocket, which was composed of an $\alpha$-helix, G262-L273, and an extended loop from N285 to H299. Energy minimisation was applied to eliminate any residual geometrical strain caused by placing WWOX $_{388-407}$ in the GSK3 $\beta$ binding pocket. Energy minimisation was achieved by performing 5000 steps of the steepest descent algorithm and 5000 steps of the conjugate gradient algorithm.

The MD simulations in this study were performed according to the standard protocol, which consists of gradual heating, equilibration and production procedures in an isothermal isobaric ensemble (NPT, $P=1$ a.t.m. and T=300 K) MD. A minimised, solvated system was used as the initial structure for subsequent MD simulations. In the 150 p.s. heating procedure, the system was gradually heated from 50 to $300 \mathrm{~K}$ at a rate of $2.4 \mathrm{~K} / 1$ p.s., followed by constant equilibration at $300 \mathrm{~K}$ for 150 p.s. After the equilibration procedure, the system underwent a 2 n.s. production procedure for conformation collection. The time step was set at 1 f.s. A snapshot was taken every 5 p.s. to record the conformation trajectory during MD production. A 12- $\AA$ cutoff was applied to treat non-bonding interactions, such as short-range electrostatic and van der Waals interactions. The particle-mesh-Ewald (PME) method was applied to treat long-range electrostatic interactions. All simulations were performed using Accelrys' Discovery Studio software with a CHARMM force field. The SHAKE algorithm was used to constrain all bonds containing hydrogen bonds to their equilibrium lengths.

Microtubule assembly assay. Microtubule assembly assays were performed by incubating tubulin $(0.2 \mathrm{mg} / \mu \mathrm{l})$ (Cytoskeleton; Denver, CO, USA) in cuvettes at $37^{\circ} \mathrm{C}$ in a thermostatic spectrophotometer and measuring the turbidity change at $350 \mathrm{~nm}$ over time. To examine the effects of WWOX and GSK3 $\beta$ on tubulin polymerisation, the GSK3 $\beta$ was pre-incubated with Tau in the presence of WWOX for $30 \mathrm{~min}$, and polymerisation was then initiated by the addition of $1 \mathrm{mM}$ GTP and followed by a light-scattering assay.

\section{Conflict of Interest}

The authors declare no conflict of interest.

1. Paige AJ, Taylor KJ, Taylor C, Hillier SG, Farrington S, Scott D et al. WWOX: a candidate tumor suppressor gene involved in multiple tumor types. Proc Natl Acad Sci USA 2001; 98 11417-11422.

2. Sze Cl, Su M, Pugazhenthi S, Jambal P, Hsu LJ, Heath J et al. Down-regulation of WW domain-containing oxidoreductase induces Tau phosphorylation in vitro. A potential role in Alzheimer's disease. J Biol Chem 2004; 279: 30498-30506.

3. Smith DI, McAvoy S, Zhu Y, Perez DS. Large common fragile site genes and cancer. Semin Cancer Biol 2007: 17: 31-41.

4. Gourley C, Paige AJ, Taylor KJ, Ward C, Kuske B, Zhang J et al. WWOX gene expression abolishes ovarian cancer tumorigenicity in vivo and decreases attachment to fibronectin via integrin alpha3. Cancer Res 2009; 69: 4835-4842.

5. Aqeilan RI, Palamarchuk A, Weigel RJ, Herrero JJ, Pekarsky Y, Croce CM. Physical and functional interactions between the Wwox tumor suppressor protein and the AP-2gamma transcription factor. Cancer Res 2004; 64: 8256-8261.

6. Chang NS, Hsu LJ, Lin YS, Lai FJ, Sheu HM. WW domain-containing oxidoreductase: a candidate tumor suppressor. Trends Mol Med 2007; 13: 12-22.

7. Doble BW, Woodgett JR. GSK-3: tricks of the trade for a multi-tasking kinase. J Cell Sc 2003; 116(Part 7): 1175-1186

8. Hedgepeth CM, Deardorff MA, Rankin K, Klein PS. Regulation of glycogen synthase kinase 3beta and downstream Wnt signaling by axin. Mol Cell Biol 1999; 19: 7147-7157.

9. Chou HY, Howng SL, Cheng TS, Hsiao YL, Lieu AS, Loh JK et al. GSKIP is homologous to the Axin GSK3beta interaction domain and functions as a negative regulator of GSK3beta. Biochemistry 2006; 45: 11379-11389.
10. Zhou F, Zhang L, Wang A, Song B, Gong K, Zhang L et al. The association of GSK3 beta with E2F1 facilitates nerve growth factor-induced neural cell differentiation. J Biol Chem 2008; 283: 14506-14515.

11. Yoshimura T, Kawano $\mathrm{Y}$, Arimura $\mathrm{N}$, Kawabata $\mathrm{S}$, Kikuchi A, Kaibuchi $\mathrm{K}$. GSK-3beta regulates phosphorylation of CRMP-2 and neuronal polarity. Cell 2005; 120: 137-149.

12. Chen ST, Chuang JI, Wang JP, Tsai MS, Li H, Chang NS. Expression of WW domaincontaining oxidoreductase WOX1 in the developing murine nervous system. Neuroscience 2004; 124: 831-839.

13. Maden M. Retinoid signalling in the development of the central nervous system. Nat Rev 2002; 3: 843-853.

14. Clagett-Dame M, McNeill EM, Muley PD. Role of all-trans retinoic acid in neurite outgrowth and axonal elongation. J Neurobiol 2006; 66: 739-756.

15. Encinas M, Iglesias M, Liu Y, Wang H, Muhaisen A, Cena V et al. Sequential treatment of SH-SY5Y cells with retinoic acid and brain-derived neurotrophic factor gives rise to fully differentiated, neurotrophic factor-dependent, human neuron-like cells. J Neurochem 2000; 75: 991-1003.

16. Hernandez F, Lucas JJ, Cuadros R, Avila J. GSK-3 dependent phosphoepitopes recognized by PHF-1 and AT-8 antibodies are present in different tau isoforms. Neurobiol Aging 2003; 24: 1087-1094

17. Gaudio E, Palamarchuk A, Palumbo T, Trapasso F, Pekarsky Y, Croce CM et al. Physical association with WWOX suppresses c-Jun transcriptional activity. Cancer Res 2006; 66: $11585-11589$.

18. Tang XN, Lo CW, Chuang YC, Chen CT, Sun YC, Hong YR et al. Prediction of the binding mode between GSK3 $\beta$ and a peptide derived from GSKIP using molecular dynamics simulation. Biopolymers 2011; 95: 461-471.

19. Dajani R, Fraser E, Roe SM, Yeo M, Good VM, Thompson V et al. Structural basis for recruitment of glycogen synthase kinase 3 beta to the axin-APC scaffold complex. EMBO J 2003; 22: 494-501.

20. Utton MA, Vandecandelaere A, Wagner U, Reynolds CH, Gibb GM, Miller CC et al. Phosphorylation of tau by glycogen synthase kinase 3 beta affects the ability of tau to promote microtubule self-assembly. Biochem J 1997; 323(Part 3): 741-747.

21. Ahmad FJ, Joshi HC, Centonze VE, Baas PW. Inhibition of microtubule nucleation at the neuronal centrosome compromises axon growth. Neuron 1994; 12: 271-280.

22. Wagner U, Utton M, Gallo JM, Miller CC. Cellular phosphorylation of tau by GSK-3 beta influences tau binding to microtubules and microtubule organisation. J Cell Sci 1996; 109 (Part 6): 1537-1543.

23. Jope RS, Yuskaitis CJ, Beurel E. Glycogen synthase kinase-3 (GSK3): inflammation, diseases, and therapeutics. Neurochem Res 2007; 32: 577-595.

24. Leroy K, Yilmaz Z, Brion JP. Increased level of active GSK-3beta in Alzheimer's disease and accumulation in argyrophilic grains and in neurones at different stages of neurofibrillary degeneration. Neuropathol Appl Neurobiol 2007; 33: 43-55.

25. Goold RG, Gordon-Weeks PR. The MAP kinase pathway is upstream of the activation of GSK3beta that enables it to phosphorylate MAP1B and contributes to the stimulation of axon growth. Mol Cell Neurosci 2005; 28: 524-534.

26. Gompel M, Soulie C, Ceballos-Picot I, Meijer L. Expression and activity of cyclin-dependent kinases and glycogen synthase kinase-3 during NT2 neuronal differentiation. NeuroSignals 2004; 13: 134-143.

27. Castano Z, Gordon-Weeks PR, Kypta RM. The neuron-specific isoform of glycogen synthase kinase-3beta is required for axon growth. J Neurochem 2011; 113: $117-130$.

28. Garnier J, Gibrat JF, Robson B. GOR method for predicting protein secondary structure from amino acid sequence. Methods Enzymol 1996; 266: 540-553.

This work is licensed under the Creative Commons Attribution-NonCommercial-No Derivative Works 3.0 Unported License. To view a copy of this license, visit http:// creativecommons.org/licenses/by-nc-nd/3.0

\section{Supplementary Information accompanies the paper on Cell Death and Differentiation website (http://www.nature.com/cdd)}

